PROCEEDINGS OF THE

AMERICAN MATHEMATICAL SOCIETY

Volume 125, Number 4, April 1997, Pages 1237-1245

S 0002-9939(97)03902-6

\title{
A NOTE ON PARACOMPACTNESS IN GENERALIZED ORDERED SPACES
}

\author{
ERIC K. VAN DOUWEN AND DAVID J. LUTZER
}

(Communicated by Franklin D. Tall)

\begin{abstract}
In this paper, we show that for generalized ordered spaces, paracompactness is equivalent to Property $\mathrm{D}$, where a space $X$ is said to have Property D if, given any collection $\{G(x): x \in X\}$ of open sets in $X$ satisfying $x \in G(x)$ for each $x$, there is a closed discrete subset $D$ of $X$ satisfying $X=\bigcup\{G(x): x \in D\}$.
\end{abstract}

\section{INTRODUCTION}

Many covering properties that are quite distinct in general spaces are equivalent to paracompactness in generalized ordered spaces. The following theorem summarizes many of the known equivalences; a more inclusive list appears in [L3]:

(1.1) Theorem. The following properties of a generalized ordered space $X$ are equivalent:

(a) $X$ is strongly paracompact.

(b) $X$ is paracompact.

(c) $X$ is metacompact.

(d) $X$ is weakly $\theta$-refinable.

(e) Every open cover of $X$ has a point-countable open refinement.

(f) $X$ is subparacompact.

(g) If $C$ is a closed subspace of $X$, then there are discrete closed sets $S$ and $T$ which are, respectively, well-ordered and reverse-well-ordered by the given ordering of $X$, have $S \cup T \subset C$, and have the property that if $x \in C$ then some points $s \in S$ and $t \in T$ have $s \leq x \leq t[\mathrm{~F}]$.

(h) Every gap and every pseudogap of $X$ is a Q-gap [GH] and [L2].

(i) No closed subspace of $X$ is homeomorphic to a stationary subset of a regular uncountable cardinal.

The purpose of this paper is to add another and quite different equivalent condition to Theorem 1.1, namely the Property D studied by van Douwen and Pfeffer $[\mathrm{vDP}]$ and by Borges and Wehrly [BW].

First, we need to recall some terminology. An open neighborhood assignment (ONA) for a subspace $Y$ of a space $X$ is a function $\mu$ from $Y$ to the open subsets

Received by the editors October 5, 1995.

1991 Mathematics Subject Classification. Primary 54F05, 54D20.

Key words and phrases. Paracompactness, Property D, generalized ordered space, linearly ordered space.

(C) 1997 American Mathematical Society 
of $X$ having $x \in \mu(x)$ for each $x \in Y$. If $A \subset Y \subset X$ and if $\mu$ is an ONA for $Y$, we will denote $\bigcup\{\mu(a): a \in A\}$ by $\mu^{*}(A)$. A topological space $\mathrm{X}$ has Property $\mathrm{D}$ if for each ONA $\mu$ for $X$, there is a discrete, closed subset $D \subset X$ having $X=\mu^{*}(D)$.

By a generalized ordered space (GO space) we mean a space that can be embedded in a linearly ordered topological space with its usual interval topology. Characterized differently, a GO space is a triple $(X, \mathcal{T},<)$ with the property that $(X, \mathcal{T})$ is a Hausdorff space and every point of $X$ has a neighborhood base whose members are convex subsets of $(X,<)[\mathrm{L} 1]$. With this terminology, we can state our main result:

(1.2) Theorem. A generalized ordered space $X$ is paracompact if and only if $X$ has Property $D$.

The easy part of that theorem appears in Section 2, and the more challenging part in Section 3. In Section 3, we will need a relative of the equivalence of (b) and (f) in Theorem 1.1. We will say that a subset $S$ of a GO space $X$ is closed to the right provided the set $\bigcup\{] \leftarrow, s]: s \in S\}$ is a closed subset of $X$, and that $S$ is closed to the left provided $\bigcup\{[s, \rightarrow[: s \in S\}$ is a closed subspace of $X$. It follows that $S$ is not closed to the right if and only if there is a limit point $r$ of $S$ having $S \subset] \leftarrow, r$. The result that we need is:

(1.3) Lemma. Suppose $X$ is a paracompact $G O$ space and $S \subset X$ is closed to the right. Then there is a closed discrete subspace $D \subset X$ that is well-ordered by the given order of $X$, has $D \subset S$, and given any $s \in S$, some $d \in D$ has $s \leq d$.

Proof. Let $T=\bigcup\{] \leftarrow, s]: s \in S\}$. Then $T$ is closed in $X$ so that, by Theorem 1.1, there is a closed, discrete subset $E \subset X$ that is well ordered by the given ordering of $X$, has $E \subset T$, and has the property that if $t \in T$ then some $e \in E$ has $t \leq e$. Clearly $T$ contains a right endpoint if and only if $S$ contains a right endpoint $r$, and in that case we let $D=\{r\}$. Hence assume that neither $T$ nor $S$ contains a right endpoint. Let $\kappa$ be the cofinality of $E$. Replacing $E$ by a suitable cofinal subset of itself if necessary, we may assume that $E=\kappa$. Let $e(0)$ be the first point of $E$. Choose $s(0)$ to be a point of $S$ with $e(0)<s(0)$. Let $e(1)$ be the first point of $E$ with $s(0)<e(1)$. An easy recursion allows us to choose points $e(\alpha) \in E$ and $s(\alpha) \in S$ for each $\alpha<\kappa$ such that if $\alpha<\alpha^{\prime}<\kappa$, then $e(\alpha)<s(\alpha)<e\left(\alpha^{\prime}\right)$. Let $D=\{s(\alpha): \alpha<\kappa\}$. Clearly $D$ is a cofinal subset of $S$, and $D$ is closed and discrete in $X$ because of the interlacing of $D$ with the closed discrete set $E$.

Questions concerning Property D. Perhaps the most interesting question about Property D concerns its relation to properties associated with paracompactness. In general spaces, Property D does not imply paracompactness: van Douwen and Pfeffer [vDP] have shown that if $S$ denotes the Sorgenfrey line, then $S \times S$ has Property D. However, we do not know whether Property D is implied by subparacompactness, paracompactness, or even the Lindelöf property (all spaces being regular and T1). Many generalized metric spaces have Property D; for example, Borges and Wehrly $[\mathrm{BW}]$ proved that all semistratifiable spaces, and all perfect inverse images of semistratifiable spaces, have Property D.

A note on authorship. Many years before Eric van Douwen died in 1987, he and Lutzer corresponded about the role of Property D in GO spaces. Lutzer observed that any GO space with Property D must be paracompact and van Douwen sketched a proof of the converse. Based upon that correspondence, Lutzer announced van 
Douwen's result in the survey article [L3], and subsequently van Douwen suggested writing a joint paper on the topic, but the proof was never written up. The heart of the current article is Section 3, below. It involves fleshing out the details of the approach that van Douwen had in mind, with a few modifications.

\section{THE EASY PART}

In this section, we use Theorem 1.1 to show that any GO space having Property $\mathrm{D}$ must be paracompact. That is the easy part of Theorem 1.2. The first lemma is proved in $[\mathrm{BW}]$.

(2.1) Lemma. If a topological space $X$ has Property $D$ and if $S$ is a closed subspace of $X$, then $S$ also has Property $D$.

(2.2) Proposition. Suppose $X$ is a GO space having Property D. Then $X$ is paracompact.

Proof. If $X$ is not paracompact, then by (1.1) there is a stationary subset $S$ of a regular uncountable cardinal $\kappa$ such that $S$ embeds in $X$ as a closed subspace. It follows from (2.1) that $S$ must have Property D. Now consider $S$ with its topology and order from the ordinal space $[0, \kappa[$. For each $x \in S, \gamma(x)=S \cap[0, x]$ is a convex ONA for $S$. According to Property $\mathrm{D}$, there is a subset $D \subset S$ that is discrete and closed in $S$ and has $S=\bigcup\{\gamma(x): x \in D\}$ so that $D$ must be a discrete, closed, cofinal subset of $S$. Then $D$ is also stationary and for each $x \in D$, there is a point $a(x)$ of $\kappa$ such that $a(x)<x$ and $D \cap] a(x), x]=\{x\}$. Apply the Pressing Down Lemma to the regressive function $a(x)$ to obtain two points $u<v$ of $D$ such that $a(u)=a(v)$. But then $a(v)<u<v$ so that $] a(v), v] \cap S$ contains both $u$ and $v$, contrary to our choice of $a(v)$. Therefore, the stationary set $S$ cannot have Property $\mathrm{D}$, and it follows that $X$ must be paracompact.

\section{THE HARD PART}

In this section, we show that any paracompact GO space has Property D by combining a sequence of lemmas. In these lemmas, a special kind of set $Y$ plays a central role. Upon first reading, it is helpful to think of $Y=X$; only in Lemma 3.6 will we need to use any other subset $Y$ of $X$.

(3.1) Definition. Given a convex ONA $\gamma$ for a GO space $X$ and a subset $Y \subset X$ with the property that $\gamma(y) \subset Y$ whenever $y \in Y$, the pair $(D, \delta)$ is $\underline{\gamma \text {-acceptable for }}$ the set $Y$ provided:

(a) $D \subset Y$ and, for each $x \in D, x \in \delta(x) \subset \gamma(x)$ and $\delta(x)$ is an open convex subset of $X$;

(b) for each $x \in D$, there are at most two points in the set $N(x)=\{z \in D-\{x\}$ : $\delta(x) \cap \delta(z) \neq \emptyset\}$

(c) for each $x \in Y$, if $\gamma(x) \cap \delta^{*}(D) \neq \emptyset$, then $x \in \delta^{*}(D)$.

(3.2) Lemma. Suppose that $(D, \delta)$ is $\gamma$-acceptable for the set $Y \subset X$. Then:

(a) $\delta^{*}(D)$ is a closed and open subset of $Y$;

(b) $D$ is a closed discrete subset of $Y$.

Proof. Being a union of the open sets $\delta(x)$ for $x \in D$, the set $\delta^{*}(D)$ is open in $X$. To see that $\delta^{*}(D)$ is closed in $Y$, suppose $p \in Y-\delta^{*}(D)$. Then $\gamma(p)$ is an open 
neighborhood of $p$, and from $(3.1)(\mathrm{c})$, we see that $\gamma(p)$ must be disjoint from $\delta^{*}(D)$. Hence $\delta^{*}(D)$ is closed in $Y$.

To see that $D$ is discrete-in-itself, suppose $d \in D$ and consider the open set $\delta(d)$. By $(3.1)(\mathrm{b})$, the set $N(d)=\{x \in D-\{d\}: \delta(x) \cap \delta(d) \neq \emptyset\}$ has at most two points, so that $U=\delta(d)-N(d)$ is an open neighborhood of $d$ with $U \cap D=\{d\}$.

Finally, $D$ is closed in $\delta^{*}(D)$ and hence also in $Y$. For suppose $x \in \delta^{*}(D)-D$. Choose $d \in D$ with $x \in \delta(d)$. Then, with $N(d)$ defined as above, $\delta(d)-(\{d\} \cup N(d))$ is a neighborhood of $x$ that is disjoint from $D$, as required.

In the next lemma, if $x \in X$ and $S \subset X$, we will write $S<x$ to mean that $s<x$ for each $s \in S$. The notation $y<S$ is similarly defined.

(3.3) Lemma. Suppose that $\gamma$ is a convex ONA for a paracompact GO space $X$ and that $Y$ is a closed and open subspace of $X$ with the property that if $y \in Y$, then $\gamma(y) \subset Y$. Fix $p \in Y$. Then there is a pair $(D, \delta)$ that is $\gamma$-acceptable for the set $Y$ and has $p \in D$.

Proof. We will first recursively define a sequence $\{B(n): n \in \omega\}$ of subsets of $Y \cap[p, \rightarrow[$ and a function $\beta$ on $B=\bigcup\{B(n): n \in \omega\}$ such that:

(1) For each $x \in B, \beta(x)$ is a convex open subset of $X$ with $x \in \beta(x) \subset \gamma(x)$ and $\beta(x) \subset Y$.

(2) $B(0)=\{p\}$ and $\beta(p)=\gamma(p)$.

(3) Each $B(n)$ is a closed discrete subspace of $X$ that is well-ordered by the given ordering $<$ of $X$.

(4) If $y \in B(n)$ and $z \in B(n+1)$, then $y<z$.

(5) If $y<z$ are both in $B$, then $\beta(y) \subset] \leftarrow, z[$ and $\beta(z) \subset] y, \rightarrow[$.

(6) If $x \geq p$ is a point of $Y$ and if $\gamma(x) \cap \beta^{*}(B) \neq \emptyset$, then $x \in \beta^{*}(B)$.

We begin by defining $B(0)=\{p\}$. By the induction hypothesis, suppose that subsets $B(0), \ldots, B(n)$ of $Y$ have already been defined in such a way that (3) and (4) in the above list are satisfied. If $B(n)$ has a greatest element $q(n)$, define $B^{\prime}(n)=\gamma(q(n))$, and if $B(n)$ has no greatest element, define $B^{\prime}(n)=B(n)$. Define a set $B^{\prime \prime}(n)$ by $B^{\prime \prime}(n)=\left\{t \in Y: t>B^{\prime}(n)\right.$ and $\left.\gamma(t) \cap B^{\prime}(n) \neq \emptyset\right\}$. We now define $B(n+1)$ as follows: if $B^{\prime \prime}(n)$ contains a right endpoint $b^{\prime \prime}$, let $B(n+1)=\left\{b^{\prime \prime}\right\}$. If $B^{\prime \prime}(n)$ contains no right endpoint and yet is closed to the right, let $B(n+1)$ be a closed discrete subset of $X$ that is well-ordered by the given ordering of $X$ and is a cofinal subset of $B^{\prime \prime}(n)$; Lemma (1.3) provides such a set because $X$ is a paracompact GO space. If $B^{\prime \prime}(n)$ is not closed to the right, then there is a unique point $r(n+1) \in X$ such that $r(n+1)$ is a limit point of $B^{\prime \prime}(n)$ and has $B^{\prime \prime}(n) \subset$ ] $\leftarrow, r(n+1)$ [. Because $Y$ is closed in $X, r(n+1) \in Y$. Because $\gamma(r(n+1))$ is an open neighborhood of $r(n+1)$, we may choose a point $e(n+1) \in \gamma(r(n+1)) \cap B^{\prime \prime}(n)$. Then $e(n+1)<r(n+1)$ and we define $B(n+1)=\{e(n+1), r(n+1)\}$. Clearly (3) and (4) are satisfied by the sets $B(0), \ldots, B(n), B(n+1)$, so that the recursion continues.

For future use, let us record several observations about the sets defined above.

Observation 1. If $B(n) \neq \emptyset$, then $B^{\prime}(n) \neq \emptyset$. However, $B^{\prime \prime}(n)$ might still be the empty set, and $B^{\prime \prime}(n)=\emptyset$ if and only if $B(n+k)=\emptyset$ for each $k>0$.

Observation 2. The set $B(n+1)$ has $0,1,2$, or infinitely many points. As noted above, the only way for $B(n+1)=\emptyset$ is for $B^{\prime \prime}(n)$ to be empty. The only way that $B(n+1)$ can have exactly one point is for that point to be the final point 
of the set $B^{\prime \prime}(n)$. The only way for $B(n+1)$ to have two points is if $B(n+1)=$ $\{e(n+1), r(n+1)\}$ in the situation where $B^{\prime \prime}(n)$ is not closed to the right. In all other cases, $B(n+1)$ must be an infinite cofinal subset of $B^{\prime \prime}(n)$.

Observation 3. The only way that $B(n+1)$ can fail to be a subset of $B^{\prime \prime}(n)$ is for $B(n+1)=\{e(n+1), r(n+1)\}$ and this occurs only when $B^{\prime \prime}(n)$ fails to be closed to the right. It follows that if $u<v$ are points of $B(n+1)$, then $u \in B^{\prime \prime}(n)$, and that the first point of $B(n+1)$ must always belong to $B^{\prime \prime}(n)$.

Observation 4. If $x \in B^{\prime \prime}(n)$, then some $y \in B(n+1)$ has $x \leq y$. This follows easily from Observation 2.

Once the sets $B(n)$ have been defined, we may define the function $\beta$ on the set $B=\bigcup\{B(n): n \in \omega\}$ as follows. For each $x \in B$, properties (3) and (4) force the set ]$\leftarrow, x] \cap B$ to be closed and discrete in $X$. Therefore the set $M(x)=X-(B-\{x\})$ is a neighborhood of $x$ in $X$. Let $\beta^{\prime}(x)$ be the convex component (with respect to the set $X)$ of the point $x$ in the set $M(x)$. Define $\beta(x)=\gamma(x) \cap \beta^{\prime}(x)$. Then $\beta(x)$ is a convex open subset of $X$ that contains $x$ and is a subset of $\gamma(x)$. Because $x \in B \subset Y$, we have $\beta(x) \subset \gamma(x) \subset Y$. Therefore property (1) holds.

As for property (2), consider the point $p \in B(0)$. We have $B^{\prime}(0)=\gamma(p)$ so that $B^{\prime \prime}(0)=\{t \in Y: t>\gamma(p)$ and $\gamma(t) \cap \gamma(p) \neq \emptyset\}$. If $B(1) \neq \emptyset$, then its first point $b(1)$ lies in $B^{\prime \prime}(0)$ according to Observation 3 so that $\gamma(p)<b(1)$. It follows that $\gamma(p) \subset \beta^{\prime}(p)$ so that $\beta(p)=\gamma(p)$. And if $B(1)=\emptyset$, then $B(n)=\emptyset$ for every $n>0$ so that $p$ is the unique point of $B$ and hence $M(p)$, defined above, is all of $X$. But then $\beta^{\prime}(p)=X$ so that once again we see that $\beta(p)=\gamma(p)$. Thus, (2) holds.

Properties (3) and (4) were built into the recursive construction of the sets $B(n)$. To verify (5), notice that if $y<z$ are points of $B$, then $X-(B-\{y\}) \subset$ ] $\leftarrow, z[\cup] z, \rightarrow[$ with the result that the convex component in $X$ of $y$ in the set $X-(B-\{y\})$ is contained in $] \leftarrow, z\left[\right.$, i.e., $\left.\beta^{\prime}(y) \subset\right] \leftarrow, z[$. But then $\left.\beta(y)=\beta^{\prime}(y) \cap \gamma(y) \subset\right] \leftarrow, z[$. Similarly, $\beta(z) \subset] y, \rightarrow[$.

Observation 5. Fix $y \in B$. If $z$ and $w$ are points of $B$ with $y<z<w$, then $\beta(y) \subset] \leftarrow, z[$ and $\beta(w) \subset] z, \rightarrow[$ so that $\beta(y) \cap \beta(w)=\emptyset$. It follows that there is at most one point $z$ of $B \cap] y, \rightarrow[$ with $\beta(y) \cap \beta(z) \neq \emptyset$. Similarly there is at most one point $z \in B \cap] \leftarrow, y[$ with $\beta(y) \cap \beta(z) \neq \emptyset$ so that the set $\{z \in B-\{y\}: \beta(z) \cap \beta(y) \neq \emptyset\}$ has at most two points. Also note that the set $\{z \in B-\{p\}: \beta(z) \cap \beta(p) \neq \emptyset\}$ has at most one member.

It remains to verify property (6) for $B$ and $\beta$, which requires several steps. Fix a point $x \in Y \cap\left[p, \rightarrow\left[\right.\right.$, and suppose that $\gamma(x) \cap \beta^{*}(B) \neq \emptyset$. For contradiction, suppose that

$$
x \text { does not belong to } \beta^{*}(B) \text {. }
$$

Claim 1. Some point $z \in B$ has $x \leq z$. For suppose not, i.e., suppose that

$$
B \subset] \leftarrow, x[\text {. }
$$

Because $\gamma(x) \cap \beta^{*}(B) \neq \emptyset$, we may choose an integer $n \in \omega$ and $b \in B(n)$ so that $\gamma(x) \cap \beta(b) \neq \emptyset$. According to $(\# \#), b<x$.

Claim 1-a. $x>B^{\prime}(n)$.

If $B^{\prime}(n)=B(n)$, this assertion follows from (\#\#), so assume that $B^{\prime}(n) \neq B(n)$. Then $B(n)$ must have a largest element $q(n)$ and $B^{\prime}(n)=\gamma(q(n))$. Note that $q(n)<x$ in light of (\#\#). If some $w \in B^{\prime}(n)$ has $x \leq w$, then convexity of $\gamma(q(n))$ 
forces $x \in \gamma(q(n))$. But then $B^{\prime \prime}(n)=\emptyset$. For if $B^{\prime \prime}(n) \neq \emptyset$, then $B(n+1) \neq \emptyset$ and, from Observation 3 above, the first point $c$ of $B(n+1)$ must belong to $B^{\prime \prime}(n)$. Hence $c$ has $c>B^{\prime}(n)$ so that $c>w$. But $c \in B(n+1) \subset B$ so that (\#\#) yields $c<x$. Combining those inequalities gives the contradiction $x>c>w \geq x$. Therefore, $B^{\prime \prime}(n)$ must be empty, and therefore $B(n+k)=\emptyset$ for all $k>0$, so that $q(n)$, known to be the final point of $B(n)$, is actually the final point of $B$. It follows that $\left[q(n), \rightarrow\left[\subset \beta^{\prime}(q(n))\right.\right.$. Because $x \in \gamma(q(n))$ as noted above, we have $x \in \gamma(q(n)) \cap \beta^{\prime}(q(n))=\beta(q(n)) \subset \beta^{*}(E)$, contrary to (\#). We now conclude that it cannot happen that $x \leq w$ for some $w \in \gamma(q(n))=B^{\prime}(n)$, and hence $x>B^{\prime}(n)$ as asserted in Claim 1-a.

Claim 1-b. $\gamma(x) \cap B^{\prime}(n) \neq \emptyset$.

If $B^{\prime}(n)=B(n)$, then $B(n)$ has no final point so that some $c \in B(n)$ has $b<c$. From (\#\#), we know $c<x$. Because $b<c$, it follows from the already established point (5) that $\beta(b) \subset] \leftarrow, c[$. Because $\gamma(x)$ is convex and meets $\beta(b)$, it follows from $c<x$ that $c \in \gamma(x) \cap B(n)=\gamma(x) \cap B^{\prime}(n)$ as required to establish Claim 1-b in the case where $B^{\prime}(n)=B(n)$.

If $B^{\prime}(n) \neq B(n)$, then $B(n)$ has a final point $q(n)$. The fact that $b \in B(n)$, combined with (\#\#), yields $b \leq q(n)<x$. If $b=q(n)$, then $\beta(b)=\beta(q(n)) \subset$ $\gamma(q(n))=B^{\prime}(n)$ so that $B^{\prime}(n) \cap \gamma(x) \supset \beta(b) \cap \gamma(x) \neq \emptyset$, as required to establish Claim 1-b. If $b<q(n)$, then Observation 5 shows that $\beta(b) \subset] \leftarrow, q(n)[$. Because $b<q(n)<x$ and $\beta(b) \cap \gamma(x) \neq \emptyset$, convexity of $\gamma(x)$ yields $q(n) \in \gamma(x)$. We conclude that $q(n)$ is a point of $\gamma(q(n)) \cap \gamma(x)=B^{\prime}(n) \cap \gamma(x)$, as required for Claim 1-b.

The combination of Claim 1-a and Claim 1-b forces $x \in B^{\prime \prime}(n)$. But then $B(n+1) \neq \emptyset$ so that from Observation 4 , some $c \in B(n+1)$ has $x \leq c$. But this is impossible in light of (\#\#) because $c \in B$. Therefore, Claim 1 is proved.

Claim 1 shows that the set $S=\{s \in B: x \leq s\}$ is nonempty. Recall that $B$ is well-ordered by the given ordering of $X$. Then let $z$ be the first element of the set $S$.

Claim 2. $x \in \beta^{\prime}(z)$.

Because of (\#), $x \notin B$ so that $x<z$. Minimality of $z$ in $S$ means that every point of the closed discrete set $B \cap$ ] $\leftarrow, z$ [ lies below $x$. Therefore we may choose a convex open set $U$ which contains the points $x$ and $z$, and has $U \cap(B \cap] \leftarrow, z[)=\emptyset$. If $z$ is the final point of $B$ (as well as the first point of $S$ ), then $x \in U \cup\left[z, \rightarrow\left[\subset \beta^{\prime}(z)\right.\right.$ as required for Claim 2. If $z$ is not the final point of $B$, let $w$ be the first point of $B$ that lies above $z$. Then $V=U \cup[z, w[$ is a convex subset of $X$ that is contained in $X-(B-\{z\})$ and that contains both $z$ and $x$. It follows that $x \in V \subset \beta^{\prime}(z)$ as required by Claim 2 .

Claim 3. $x \in \gamma(z)$.

Choose $k$ so that $z \in B(k)$. Because $p \leq x<z$, we know that $z \notin B(0)$ so that $k \geq 1$ and the set $B(k-1)$ is defined and nonempty.

If $\gamma(z) \cap B \cap] \leftarrow, z[\neq \emptyset$, choose $c \in \gamma(z) \cap B$ with $c<z$. Minimality of $z$ in the set $S$ yields $c<x<z$. Convexity of $\gamma(z)$ then yields $x \in[c, z] \subset \gamma(z)$ as required by Claim 3. Therefore, suppose

$$
(\# \# \#) \quad \gamma(z) \cap B \cap] \leftarrow, z[=\emptyset .
$$

Then $z$ must be a point of $B^{\prime \prime}(k-1)$ because, by Observation 3 , the only way that $z \notin B^{\prime \prime}(k-1)$ is if $B^{\prime \prime}(k-1)$ is not closed to the right and $z$ is its right endpoint, 
denoted by $r(k)$ in the construction of the sets $B(n)$. But in the case there is a point $e(k) \in \gamma(z) \cap B^{\prime \prime}(k-1)$ with $e(k)<z$, then $e(k) \in B(k) \subset B$, contrary to (\#\#\#). Thus, $z \in B^{\prime \prime}(k-1)$ so that $z>B^{\prime}(k-1)$ and $B^{\prime}(k-1) \cap \gamma(z) \neq \emptyset$. Because $\gamma(z)$ contains no point of $B$ lying below $z$ (according to (\#\#\#)), it follows that $B^{\prime}(k-1) \neq B(k-1)$. Therefore $B(k-1)$ has a largest point $q=q(k-1)$ and $B^{\prime}(k-1)=\gamma(q)$. Because $q \in B(k-1), q<z$. Then minimality of $z$ in $S$ yields $q<x<z$. We claim that $z$ must be the first point of $B(k)$. For suppose some $w \in B(k)$ has $w<z$. By Observation 3, $w \in B^{\prime \prime}(k-1)$ so that $w>B^{\prime}(k-1)=\gamma(q)$. Because $q<z$ and $\gamma(q) \cap \gamma(z) \neq \emptyset$, convexity forces $w \in[q, z] \subset \gamma(q) \cup \gamma(z)$. But $w>\gamma(q)$ so that $w \in \gamma(z)$, showing that $\gamma(z)$ contains a point of $B \cap] \leftarrow, z[$, contrary to (\#\#\#). Therefore, $z$ must be the first point of $B(k)$. As a result, $q$ and $z$ are adjacent points in the set $B$, with $q$ being the last point of $B(k-1)$ and $z$ being the first point of $B(k)$. Therefore $x \in] q, z\left[\subset \beta^{\prime}(q) \cap \beta^{\prime}(z)\right.$. But we also know that $\gamma(q) \cap \gamma(z) \neq \emptyset$ so that convexity of $\gamma$ yields $x \in] q, z[\subset \gamma(q) \cup \gamma(z)$. If $x \in \gamma(q)$, then $x \in \gamma(q) \cap \beta^{\prime}(q)=\beta(q)$, and if $x \in \gamma(z)$, then $x \in \gamma(z) \cap \beta^{\prime}(z)=\beta(z)$. Each of these possibilities contradicts (\#) so that Claim 3 is established.

Combining Claim 2 and Claim 3, we conclude that $x \in \beta^{\prime}(z) \cap \gamma(z)=\beta(z) \subset$ $\beta^{*}(B)$, and this contradicts (\#). Therefore, (6) is established.

In an entirely analogous fashion, we find sets $A(n) \subset Y \cap] \leftarrow, p]$ and a function $\alpha$ defined on the set $A=\bigcup\{A(n): n \in \omega\}$ such that:

$\left(1^{\prime}\right)$ For each $x \in A, \alpha(x)$ is a convex open subset of $X$ with $x \in \alpha(x) \subset \gamma(x)$ and $\alpha(x) \subset Y$.

$\left(2^{\prime}\right) \quad A(0)=\{p\}$ and $\alpha(p)=\gamma(p)$.

$\left(3^{\prime}\right)$ Each $A(n)$ is a closed discrete subspace of $X$ that is reverse-well-ordered by the given ordering of $X$.

$\left(4^{\prime}\right)$ If $y \in A(n)$ and $z \in A(n+1)$, then $z<y$.

$\left(5^{\prime}\right)$ If $y<z$ are both in $A$, then $\left.\alpha(y) \subset\right] \leftarrow, z[$ and $\alpha(z) \subset] y, \rightarrow[$.

(6') If $y \leq p$ is a point of $Y$ and if $\gamma(y) \cap \alpha^{*}(A) \neq \emptyset$, then $y \in \alpha^{*}(A)$.

Now define $D=A \cup B$ and define $\delta(x)=\alpha(x)$ if $x \in A$, and $\delta(x)=\beta(x)$ if $x \in B$. Because $A \cap B=\{p\}$, properties (2) and (2') show that $\delta$ is well-defined.

It remains to verify that the pair $(D, \delta)$ is $\gamma$-acceptable for the set $Y$. That $(D, \delta)$ satisfies part (a) of Definition 3.1 is clear. To verify part (b), observe that if $x \in A$ and $y \in B$ are both distinct from $p$, then $\delta(x) \cap \delta(y)=\alpha(x) \cap \beta(y) \subset] \leftarrow, p[\cap$ ]$p, \rightarrow[=\emptyset$. For points of the set $D-\{p\}$, part (b) now follows from (5) and (5') of the proof above. It remains to consider the point $p$. Properties $(5)$ and $\left(5^{\prime}\right)$ show that there is at most one point $x \in(A-\{p\})$ and at most one point $y \in(B-\{p\})$ such that $\delta(x) \cap \delta(p) \neq \emptyset \neq \delta(p) \cap \delta(y)$, as required by part (b) of (3.1). Finally suppose that $y \in Y$ and $\gamma(y) \cap \delta^{*}(D) \neq \emptyset$. Because $\delta^{*}(D)=\alpha^{*}(A) \cup \beta^{*}(B)$, it follows from (6) and $\left(6^{\prime}\right)$ that $y \in \alpha^{*}(A) \cup \beta^{*}(B)=\delta^{*}(D)$. Therefore, $(D, \delta)$ is a $\gamma$-acceptable pair for $Y$.

(3.4) Definition. For any convex ONA $\gamma$ on $X$, let $\mathcal{P}(\gamma)=\{(E, \mu):(E, \mu)$ is a $\gamma$-acceptable pair for the set $X\}$. Define $\prec$ on the set $\mathcal{P}(\gamma)$ by the rule that $(E, \mu) \prec(F, \nu)$ provided $E \subset F$ and $\mu$ and $\nu$ agree on the set $E$.

(3.5) Lemma. $\mathcal{P}(\gamma)$ has a maximal element.

Proof. Apply Lemma 3.3 with $Y=X$ to see that $\mathcal{P}(\gamma)$ is not empty. It is easy to check that any linearly ordered subcollection of $\mathcal{P}(\gamma)$ has an upper bound in $\mathcal{P}(\gamma)$ so that Zorn's lemma provides the required maximal element. 
(3.6) Lemma. If $(E, \mu)$ is a maximal element of $\mathcal{P}(\gamma)$, then $\mu^{*}(E)=X$.

Proof. Suppose not. Fix $p \in X-\mu^{*}(E)$. Because $(E, \mu)$ is an acceptable pair, it follows that $\gamma(p) \cap \mu^{*}(E)=\emptyset$. Let $Y$ be the set $Y=\bigcup\{\gamma(t): t \in X$ and $\gamma(t) \cap$ $\left.\mu^{*}(E)=\emptyset\right\}$. Then $p \in Y$.

We claim that $Y$ has the special properties described in the hypothesis of Lemma 3.3. First suppose $y \in Y$. Choose $t \in X$ such that $\gamma(t)$ is disjoint from $\mu^{*}(E)$ and such that $y \in \gamma(t)$. If $\gamma(y) \cap \mu^{*}(E) \neq \emptyset$, then we would have $y \in \mu^{*}(E)$ so that $y \in \gamma(t) \cap \mu^{*}(E)=\emptyset$. Hence, $\gamma(y) \cap \mu^{*}(E)=\emptyset$ so that $\gamma(y)$ is one of the sets used to form $Y$ and $\gamma(y) \subset Y$.

We next claim that $Y=X-\mu^{*}(E)$. First suppose $y \in Y$. As noted above, $\gamma(y) \cap \mu^{*}(E)=\emptyset$ so that $y \in X-\mu^{*}(E)$. Next suppose $z \in X-\mu^{*}(E)$. Then $\gamma(z) \cap \mu^{*}(E)=\emptyset$ because $(E, \mu)$ is acceptable so that $z \in \gamma(z) \subset Y$. Hence, $Y=X-\mu^{*}(E)$ so that $Y$ is both open and closed in $X$ (because of Lemma 3.2(a)).

Now apply Lemma 3.3 to find a pair $(D, \delta)$ that is $\gamma$-acceptable for the set $Y$. Let $F=D \cup E$ and define $\nu$ on $F$ by $\nu(x)=\mu(x)$ if $x \in E$ and $\nu(x)=\delta(x)$ if $x \in D$. Because $D \cap E \subset Y \cap \mu^{*}(E)=\emptyset, \nu(x)$ is well-defined.

We claim that $(F, \nu)$ is $\gamma$-acceptable for the set $X$. Part (a) of Definition 3.1 is clearly satisfied. To verify part (b), observe that if $y \in D$ and $z \in E$, then $\nu(y)=\delta(y) \subset \gamma(y) \subset Y$ and $\nu(z)=\mu(z) \subset \mu^{*}(E)$ so that $\nu(y) \cap \nu(z)=\emptyset$. Therefore, if $y \in D$ and $z \in F$ have $\nu(y) \cap \nu(z) \neq \emptyset$, then $z \in D$. Because $(D, \delta)$ is acceptable, there are at most two such points $z$ in the set $D-\{y\}$. Therefore there are at most two such points in $F-\{y\}$. A similar argument applies if $y \in E$. Therefore, part (b) of 3.1 is verified.

To verify part (c) of Definition 3.1, suppose that $x \in X$ and that $\gamma(x)$ meets $\nu^{*}(F)$. Then either $\gamma(x) \cap \mu^{*}(E) \neq \emptyset$ or else $\gamma(x) \cap \delta^{*}(D) \neq \emptyset$. In the first case, because $(E, \mu)$ is $\gamma$-acceptable for the set $X$, we know that $x \in \mu^{*}(E) \subset \nu^{*}(F)$, as required. Therefore, assume that $\gamma(x) \cap \mu^{*}(E)=\emptyset$. But then $x \in Y$ and $\gamma(x) \cap \delta^{*}(D) \neq \emptyset$, so that because $(D, \delta)$ is $\gamma$-acceptable for the set $Y$ we know that $x \in \delta^{*}(D) \subset \nu^{*}(F)$, as required.

We have now proved that $(F, \nu) \in \mathcal{P}(\gamma)$. Because $p \in F-E,(E, \mu)$ lies strictly below $(F, \nu)$ in the ordering of $\mathcal{P}(\gamma)$, which is impossible because $(E, \mu)$ is a maximal element of $\mathcal{P}(\gamma)$. This contradiction establishes that $\mu^{*}(E)=X$, as claimed.

(3.7) Proposition. Any paracompact GO space has Property D.

Proof. Let $\gamma$ be any ONA for the paracompact GO space $X$. Replacing $\gamma(x)$ by its convex component that contains $x$ if necessary, we may assume that each $\gamma(x)$ is convex in $X$. Use Lemma 3.3 to find a maximal element $(E, \mu) \in \mathcal{P}(\gamma)$. According to Lemma 3.6, $\mu^{*}(E)=X$ and from Lemma 3.2(a), $E$ is a closed discrete subspace of $X$. But then $\mu(x) \subset \gamma(x)$ for each $x \in X$ and therefore $X=\bigcup\{\gamma(x): x \in E\}$, as required.

\section{REFERENCES}

[BW] Borges, C. and Wehrly, A., A study of D-spaces, Topology Proceedings, 16 (1991), 7-15. MR 94a:54059

[vDP] van Douwen, E. and Pfeffer, W., Some properties of the Sorgenfrey line and related spaces, Pacific J. Math., 81 (1979), 371-377. MR 80h:54027

[F] Faber, M.J., Metrizability in generalized ordered spaces, Mathematical Centre Tracts, no.53, Amsterdam, 1974. MR 54:6097

[GH] Gillman, L., and Henricksen, M., Concerning rings of continuous functions, Trans. Amer. Math. Soc., 77 (1954), 340-362. MR 16:156g 
[L1] Lutzer, D., On generalized ordered spaces, Dissertationes Math., vol. 89, 1971. MR 48:3018

[L2] Lutzer, D., Ordinals and paracompactness in ordered spaces, Proc. Second Pittsburgh Conference, December 1972, Springer Lecture Notes in Mathematics 378, pp. 258-266. MR 50:14692

[L3] Lutzer, D., Ordered topological spaces, Surveys in General Topology, ed. by G.M. Reed, Academic Press, 1980, pp. 247-295. MR 81d:54026

Department of Mathematics, Ohio University, Athens, Ohio 45701

Department of Mathematics, College of William and Mary, Williamsburg, Virginia 23187

E-mail address: DJLUTZ@MAIL.WM.EDU 